\title{
Correlation of the angular distributions of the antisymmetrical component of the microwave backgrounds temperature deviation and the redshifts of quasars
} Kudriavtcev Iu.

Saint-Petersburg, Russia;

E-mail: Kudriavtcev<juku@bk.ru>;

We checked the assumption of possible correlation of the antisymmetrical component of the microwave backgrounds temperature deviation and the redshifts of quasars. We present the results of the comparative analysis of the microwave background distributions and the redshifts of quasars, confirming the existence of the assumed phenomena.

We discovered the positive correlation of the distributions of the antisymmetrical component of the microwave backgrounds temperature deviation and the angular distributions of the average values of redshifts of quasars from SDSS-DR7 catalog in the areas of the celestial sphere extending up to 120 degrees with Pearson correlation coefficient till +0.75 . We noted the negative correlation of the angular distributions of the average values of redshifts of quasars in the opposite areas of the celestial sphere in the areas of the celestial sphere extending up to 90 degrees with the value of Pearson correlation coefficient till -0.59.

These results confirm the need for a more careful study of the phenomenon of the central symmetry of the celestial sphere, which shows inconsistency of the standard cosmological model and deriving from it conclusions about the dynamics of the universe.

Keywords: microwave background, redshift, quasar.

DOI: $10.18698 / 2309-7604-2015-1-248-265$

\section{Introduction}

Any variable on the spherical surface, including the microwave background temperature, can be presented as a sum of the symmetrical and antisymmetrical components, representing halfsum and half-difference of its values in diametrically opposite points of the sphere.

Discovered in [1] symmetry of the large-scale microwave background inhomogeneities was considered by the authors as axis symmetry of the Universe, i.e. the existence of the symmetry axis defined in the space, which contradicts to the space isotropy principle in the foundation of the Relativity Theory. However in [2] it was shown that this characteristic of the microwave background can be interpreted not as an axis symmetry but as a central one, or actually a central antisymmetry, when each inhomogeneity of the microwave background temperature corresponds to an analogous inhomogeneity of the opposite sign in the opposite, i.e. centrally symmetrical point of the celestial sphere. At that, in [2] it was shown that on the celestial sphere two types of central symmetry manifest at ones - one with positive and one with negative signs. Central symmetry of 
the celestial sphere manifests also in certain particularities of the mutual disposition and luminosity of the quasars [3].

The phenomenon of the central symmetry is naturally interpreted in the modified cosmological model [4],[5] built on the metric considering the non-zero value of the scale factor differential in the expanding Universe. Taking it into account when drawing the metric tensor leads to the change of the time component $\mathrm{g}_{00}$ and to the model that describes the closed Universe with a slower development dynamics than a standard Big Bang model. Its lifetime appears to be enough for a light beam radiated by the source to travel around the whole closed Universe. At that, new effects caused by the opportunity to observe the signals from the same source it two opposite points of the celestial sphere, become possible, which causes the central symmetry.

This article is dedicated to the confirmation of the following from the model assumption that the distribution of the antisymmetrical component of microwave background temperature deviation can correlate with the angular distribution of the average values of redshift of quasars.

\section{Relation of the microwave background inhomogeneities to the initial temperature inhomogeneities and matter movement radial velocities at the hydrogen recombination period}

The dependency of the frequency of the received thermal radiation maximum $\mathrm{F}$ from the source movement velocity is given by the formula of relativistic Doppler effect

$$
F=f_{0}\left(1-\beta^{2}\right)^{1 / 2} /(1+\beta \cos \vartheta)
$$

where $f_{0}$ - thermal radiation maximum frequency of the source, $\beta$ - relative source velocity, $\vartheta-$ the angle between the movement velocity and the eyebeam.

At $\beta<<1$ and $|\cos \vartheta|=1$ we obtain

$$
F \approx f_{0}(1+\beta)
$$

The observed temperature of the background $T$ is related to the temperature $T_{z}$ during the hydrogen recombination epoch (transparency epoch) by the expression

$$
T_{z}=T(1+Z)
$$


Where $\mathrm{Z}$ - redshift at the moment of transparency.

For a moving source we will obtain

$$
T=T_{z}(1 \pm \beta) /(1+Z)
$$

Symmetrical and antisymmetrical components of the microwave background temperature deviation are defined by the expressions:

$$
\begin{aligned}
& t_{\text {symm }}=\left(t_{1}+t_{2}\right) / 2 ; \\
& t_{\text {asymm }}=\left(t_{1}-t_{2}\right) / 2 ;
\end{aligned}
$$

where $t_{1}$ and $t_{2}$ - deviations of the microwave background temperature deviations in the considered and opposite (centrally symmetrical) points of the celestial sphere, related to the absolute temperatures $T_{1}$ and $T_{2}$ by the expression $t=T-T_{0}$, where $T_{0}-$ average value of the temperature. From where

$$
\begin{gathered}
t_{\text {symm }}=\left(T_{1}+T_{2}\right) / 2-T_{0} ; \\
t_{\text {asymm }}=\left(T_{1}-T_{2}\right) / 2 ;
\end{gathered}
$$

According to the modified cosmological model we can observe the same radiation source in two opposite points of the celestial sphere - in one of them by the signal that came to us straight and in the other one by the signal that came to us from the opposite side around the whole closed Universe. At that, if in one of them the radial velocity of the source is directed to us $(+\beta)$, in another one it will be directed from us (- $\beta$ ). From that, suppressing $\mathrm{Z}$ for simplicity, for the source moving with relative velocity $\beta$, we will obtain

$$
t_{\text {symm }}=(1 / 2)[T(1+\beta)+T(1-\beta)]-T_{0}=T-T_{0}
$$




$$
t_{\text {asymm }}=(1 / 2)[T(1+\beta)-T(1-\beta)]=T \beta
$$

This way the symmetrical component of the temperature deviation $t_{\text {symm }}$ appears to be proportional to the initial temperature deviation in the observed area of the space, and antisymmetrical component $t_{\text {asymm }}$ is proportional to the radial velocity of the matter movement in this area.

Of course the microwave background observed in some point of the celestial sphere did not come to us from a certain point of the space but is a sum of radiations emitted by different areas of the matter located on different distances along the eyebeam. However if the result of these signals corresponds to the positive velocity in the direction of the eyebeam which according to (10) corresponds to $t_{a s y m m}>0$, we can assume that the initial masses of the matter at the recombination epoch were moving along this eyebeam mostly towards us. This could have led to the situation where the observed objects that were formed later out of this matter are located in an averagely less distances from us than the objects located in those distances for which $t_{\text {asymm }}<0$, and the initial masses of matter were moving in these directions mostly from us.

\section{Initial data for analysis}

Out of the analysis of the dependencies of the luminosities of quasars and galaxies from the redshift and their interpretation in the modified cosmological model [5] it follows that the value of $\mathrm{Z} \approx 2$ approximately corresponds to the complete circle that the signal makes around the closed Universe. From that, as we can observe the objects located at the values of $\mathrm{Z}$, reaching and exceeding 2, we can see the Universe all the way down.

To compare the observed objects with the characteristics of the microwave background that came to us from all the possible depths of the Universe, we should choose the objects located on all possible distances, i.e. all the sections of the Big Circle of the closed Universe. It is easy to see the galaxies do not meet this requirement. The main amount of the galaxies included to catalogs has values of $\mathrm{Z}<0.3$, contrary to the quasars, the main amount of which is at $\mathrm{Z}>0.3$. According to the evaluation above, we can think that the signals from quasars as well as the microwave background, come to us from all the depths of the Universe. That is why the data we used for analysis is from SDSS-DR7 catalog [6], that includes more than 105 thousands of quasars.

For the numerical analysis of the microwave background temperature deviation we have used the data obtained from the file in FITS format (wmap_ilc_7yr_v4.fits) from the web site [7]. 
The microwave background data exists for all the areas of the celestial [7], while the data of distribution and characteristics of quasars exists only for the observation angles free of screening effect of our Galaxy which limits the area of angles suitable for the comparative analysis of the angular distributions. On the Picture 1 we show the distribution in this area of the quasars from SDSS-DR7 catalog in the galactic coordinate system where the microwave background distribution is shown as well.

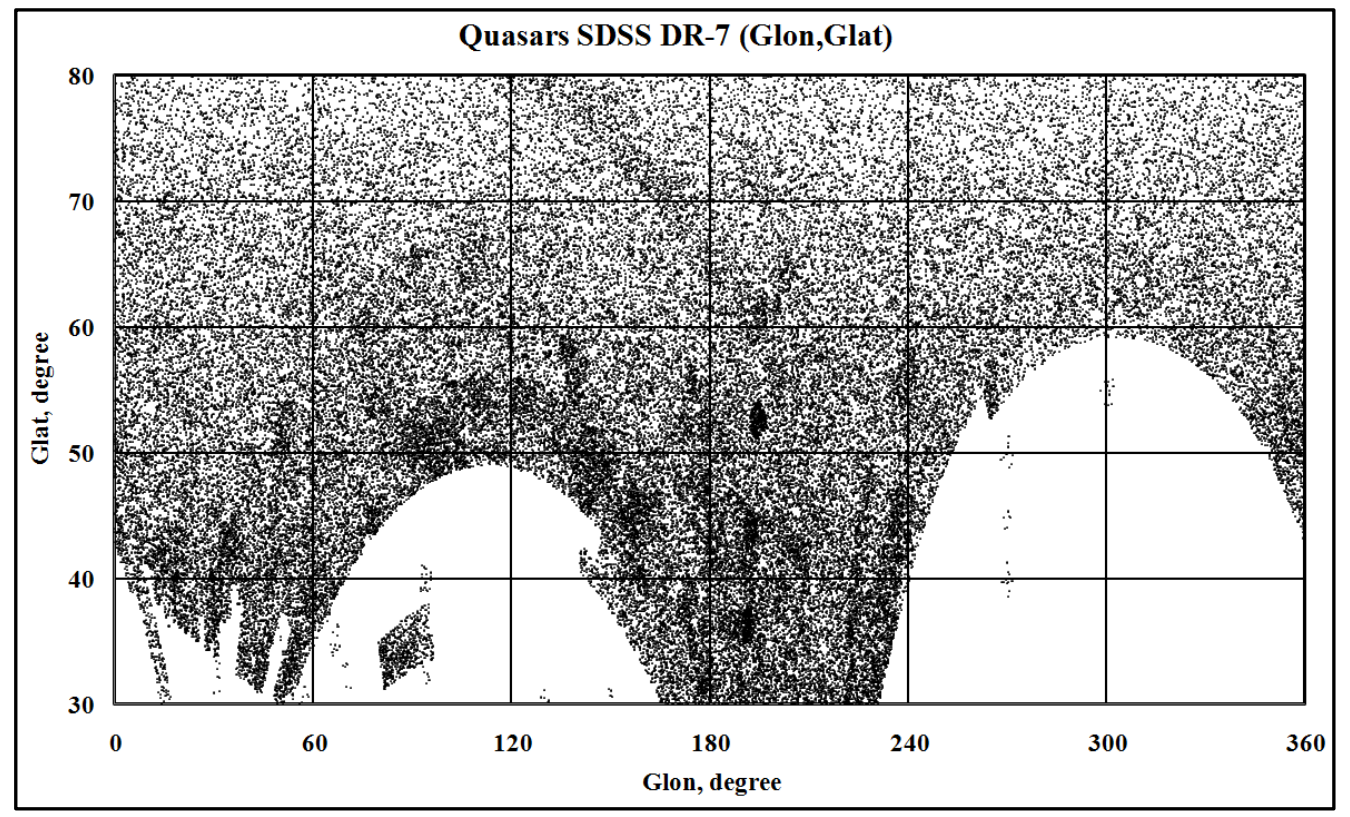

Fig. 1. Distribution of the quasars from SDSS-DR7 catalog on the available for the comparative analysis area of the celestial sphere in the galactic coordinates

On the Picture 2 we show the distribution around the celestial sphere of the microwave background deviations antisymmetrical component, calculated from the data of wmap_ilc_7yr_v4.fits by the formula (6). It is shown in Mollweide homalographic projection. The area of the celestial sphere suitable for comparative analysis is marked by a rectangle. 


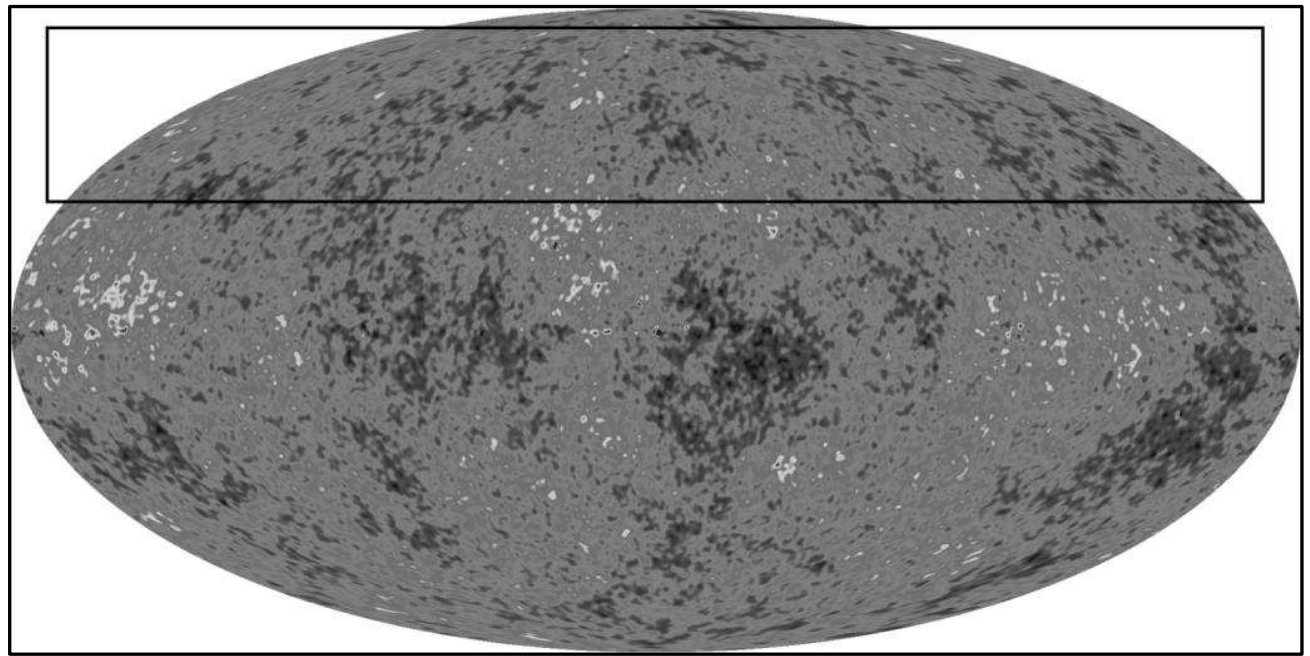

Fig. 2. Distribution on the celestial sphere of the antisymmetrical component of the microwave background deviations calculated from the data of wmap_ilc_7yr_v4.fits by the formula (6). Mollweide homalographic projection.

Out of the data of the antisymmetrical component of the microwave background shown on the Picture 2 we calculated its distributions in the galactic coordinates Glon, Glat in the suitable for analysis area (Picture 1.) with step $2^{0} \times 2^{0}$. As the positive values of the antisymmetrical component (Asymm) correspond to the velocity directed towards the observer, i.e. its increase corresponds to the expected decrease of the average remoteness of the observed objects, the obtained distribution for the comparative analysis was taken with the opposite sign (-Asymm).

From the SDSS-DR7 date in the same area of the angular coordinates with the same step $2^{0} \times 2^{0}$ we calculated the average values of $\mathrm{Z}$, i.e. the arithmetical averages of $\mathrm{Z}$ of all the quasars from the catalog, the angular coordinates of which are located within the limits of the corresponding cell $\left(2^{0} \times 2^{0}\right)$. Total amount of quasars in the area is 81517 . The amount of the quasars in the cell varies from 0 to 167,17 quasars per 1 cell on the average. The small averaging base determined a very big unevenness of the obtained averaged values Zmean, varying within the period from 0,8 to 2,8 . The average value throughout the area is 1,57 .

To decrease the noise component both distributions described above «Asymm(Glon;Glat)» and «Zmean(Glon;Glat)» were double smoothed in an identical way. First for every cell with $\left(\right.$ Glon $_{0} ;$ Glat $\left._{0}\right)$ coordinates we calculated the value average by 5 neighboring cells in a direction Glon, i.e. by the range $\left(\right.$ Glon $_{0}-4^{0}<$ Glon $<$ Glon $\left._{0}+4^{0}\right)$, and then the obtained data was averaged once again by a wider range $\left(\right.$ Glat $_{0}-2^{0}<$ Glat $\left.^{2} \mathrm{Glat}_{0}+2^{0} ; \mathrm{Glon}_{0}-4^{0}<\mathrm{Glon}<\mathrm{Glon}_{0}+4^{0}\right)$, i.e. by the rectangular area sized $3 \times 5$ cells $\left(6^{0} \times 10^{0}\right)$. 
The results of the comparative analysis of the angular distributions of the antisymmetrical component of the microwave background deviations and average quasars redshifts

The distributions -Asymm(Glon;Glat) and Zmean(Glon;Glat), obtained as the result of the double smoothing described above are shown on Picture 3 and Picture 4. The interval of the change of the distribution -Asymm is from -87 to $+79 \mathrm{mmK}$, and the interval of the change of Zmean is from 1,35 to 1,75 .

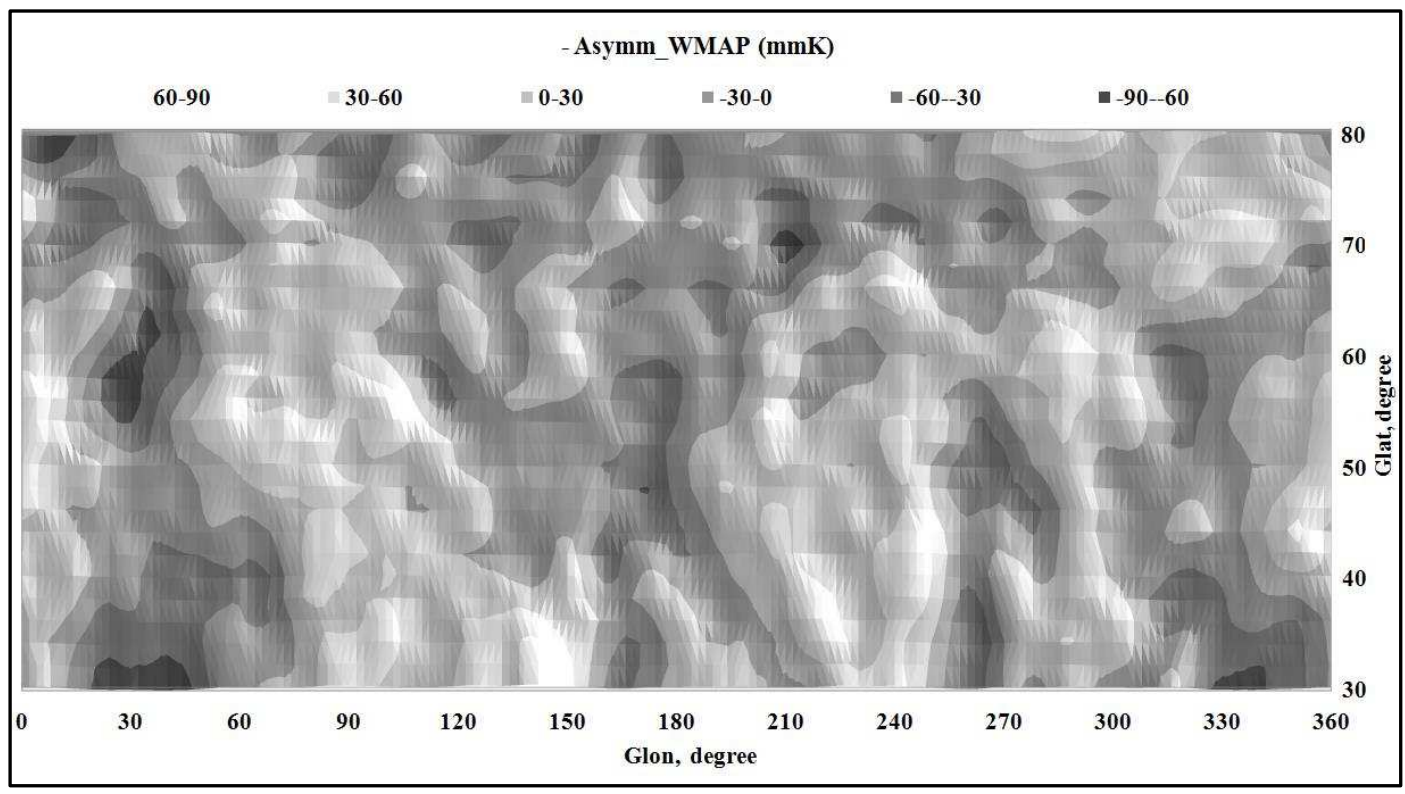

Fig. 3. Distribution of the microwave background assymetrical component deviations Asymm(Glon;Glat) throughout the celestial sphere.

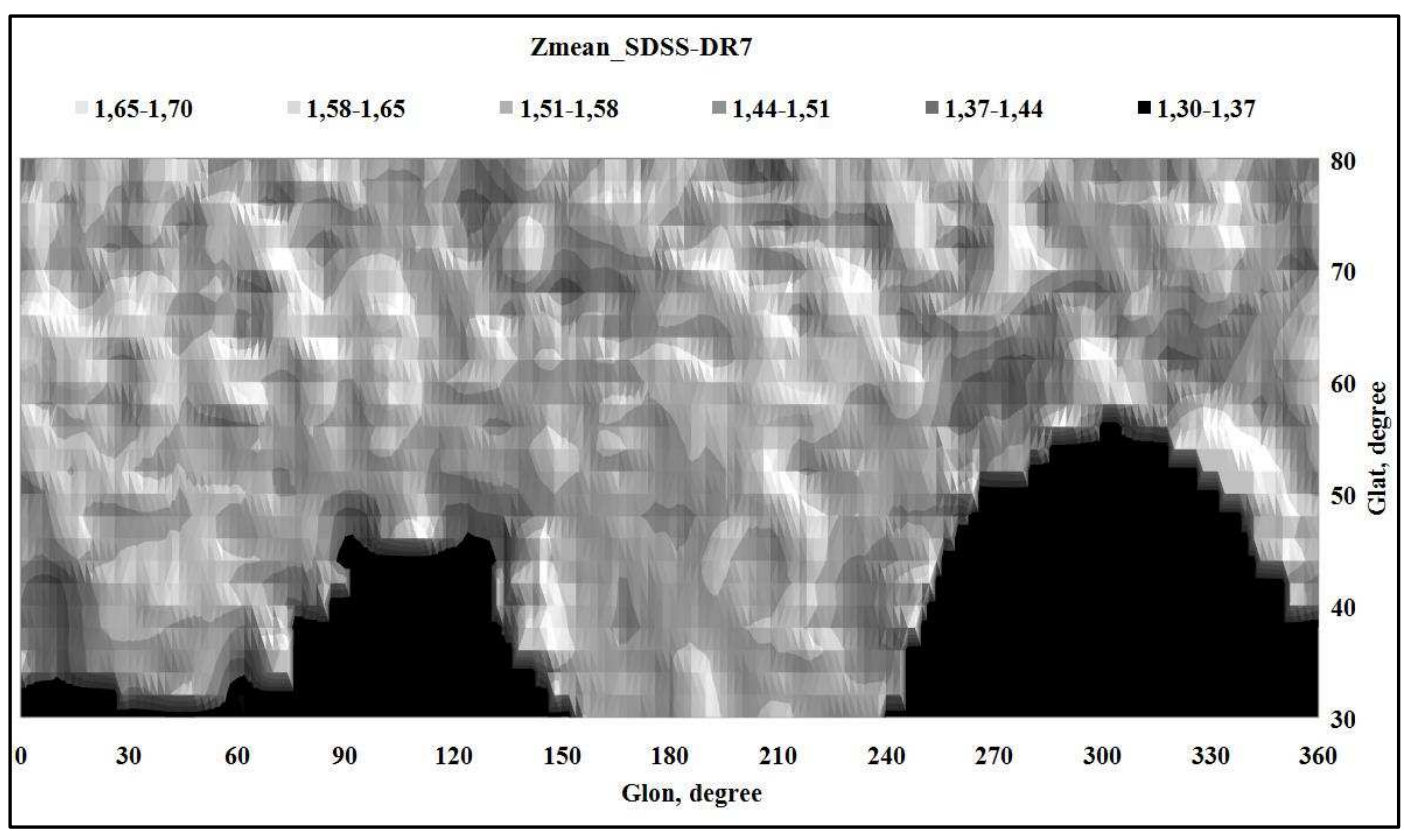


Fig. 4. Distribution of the average values Zmean(Glon;Glat) of the redshifts of quasars from the SDSS-DR7 catalog throughout the celestial sphere.

We should note that we compare the signals radiated at the interval of billions of years, so it is difficult to expect the exact correlation of their angular distributions if no for other reason than because the initial masses of the matter could have had, apart from radial velocities, also the tangential velocities that could have led to a significant shift from the moment of radiation that we now consider microwave background till the moment of radiation by the objects formed from it (quasars).

However even an initial visual analysis of the shown 2-dimensional distributions discovers the visible elements of a large-scale correlation. At that, some of the similar to each other elements of the compared distributions are shifted in relation to each other. In the Glon direction the visible shifts reach $10-25$ degrees. We can assume that these shifts are determined by the mentioned above influence of the tangential velocities of the corresponding masses movement.

To decrease the influence of these shifts we performed the smoothing of the distributions by a wider interval of the angular coordinates: on the second stage the data is averaged by the interval (Glat $0^{-} 4^{0}<$ Glat $<$ Glat $_{0}+4^{0}$; Glon $_{0}-14^{0}<$ Glon $<$ Glon $_{0}+14^{0}$ ), i.e. by the rectangular area in size $5 \times 15$ cells $\left(10^{0} \times 30^{0}\right)$. At that, the interval of the change of the obtained values -Asymm is from -50 to $+42 \mathrm{mmK}$, and the interval of the change of Zmean is from 1,45 to 1,67.

Obtained after the expanded averaging 2-dimentional diagrams are analogous to the ones shown above and are not presented here. On the Pictures 5,6 we show the results of the comparison of the graphs of distributions of these values by the axis Glon, on the Picture 7 - by the axis Glat. For the alignment of the curves for each of the shown values graphs pairs the values of -Asymm were transformed by the law: «-WMAP» $=-$ Asymm*0.002 + 1.4.

On the Picture 5 we show the pairs of curves for the same value Glat $=56^{0}$ at different smoothing modes. When smoothing by the rectangle $6^{0} \times 10^{0}$ the large-scale correlation of the curves is more obvious but the small-scale inhomogeneities lead to the decrease of Pearson correlation coefficient. All the curves shown on the Pictures 6,7, are smoothed by the rectangle $10^{0} \times 30^{0}$.

On all the shown pairs of curves we can see the correlation with a characteristic scale from 30 to 120 degrees. On the curves from the Picture 6 we can see that the correlations are more significant on the interval Glon $<180^{\circ}$.

The values of the calculated Pearson correlation coefficient Rxy are shown for the curves of the Pictures 5, 7 on the graphs, for the curves of the Picture 6 -in the Table 1 . 
Table 1. Pearson correlation coefficient values Rxy for the curves from the Picture 6.

\begin{tabular}{|c|c|c|c|}
\hline Glat, degree: & $\mathbf{6 0}$ & $\mathbf{7 2}$ & $\mathbf{7 4}$ \\
\hline $\mathbf{R x y}(\mathbf{0}<$ Glon<360) & $-0,02$ & 0,34 & 0,19 \\
\hline $\mathbf{R x y}(\mathbf{0}<$ Glon< $<\mathbf{1 8 0})$ & 0,31 & $\mathbf{0 , 5 6}$ & $\mathbf{0 , 6 3}$ \\
\hline $\mathbf{R x y}(\mathbf{1 8 0}<$ Glon<360) & 0,00 & 0,16 & $-0,22$ \\
\hline
\end{tabular}

The data from the Table 1 confirms that the distributions correlation is more significant on the interval Glon $<180^{\circ}$, where the correlation coefficient reaches 50-60\%. At that, on the interval $180^{\circ}<$ Glon $<360^{\circ}$ Rxy decreases down to zero or even negative values.

For a more detailed research of the distributions correlation we calculated the values of Pearson correlation coefficient for rectangular sections located in different places of the area available for comparison.

On the Picture 8 we show the location in the studied area of the rectangular sections sized $18^{0} \times 90^{0}$ (9x45 cells) with maximal by module values of Rxy. We see that in two sections the value of the correlation coefficient of 2-dimensional distributions -Asymm(Glon;Glat) and Zmean(Glon;Glat) reaches values 0.7 and 0.75 .

The location of the areas of high positive and negative correlation is the same for wider areas with sizes $18^{0} \times 120^{\circ}$ (9x60 cells). For them on the areas $57^{\circ}<$ Glat $<75^{0}$ the maximal positive value of Rxy is reached at $50^{\circ}<$ Glon $<170^{\circ}$ and makes 0.64 , and the negative is reached at $190^{\circ}<$ Glon $<310^{\circ}$ and makes -0.45 . The value of Rxy for the section located between the unavailable for comparison areas at small values of Glat, in this case cannot be determined as the size of the section exceeds the distance between the edges of unavailable areas (about $100^{\circ}$ ). 

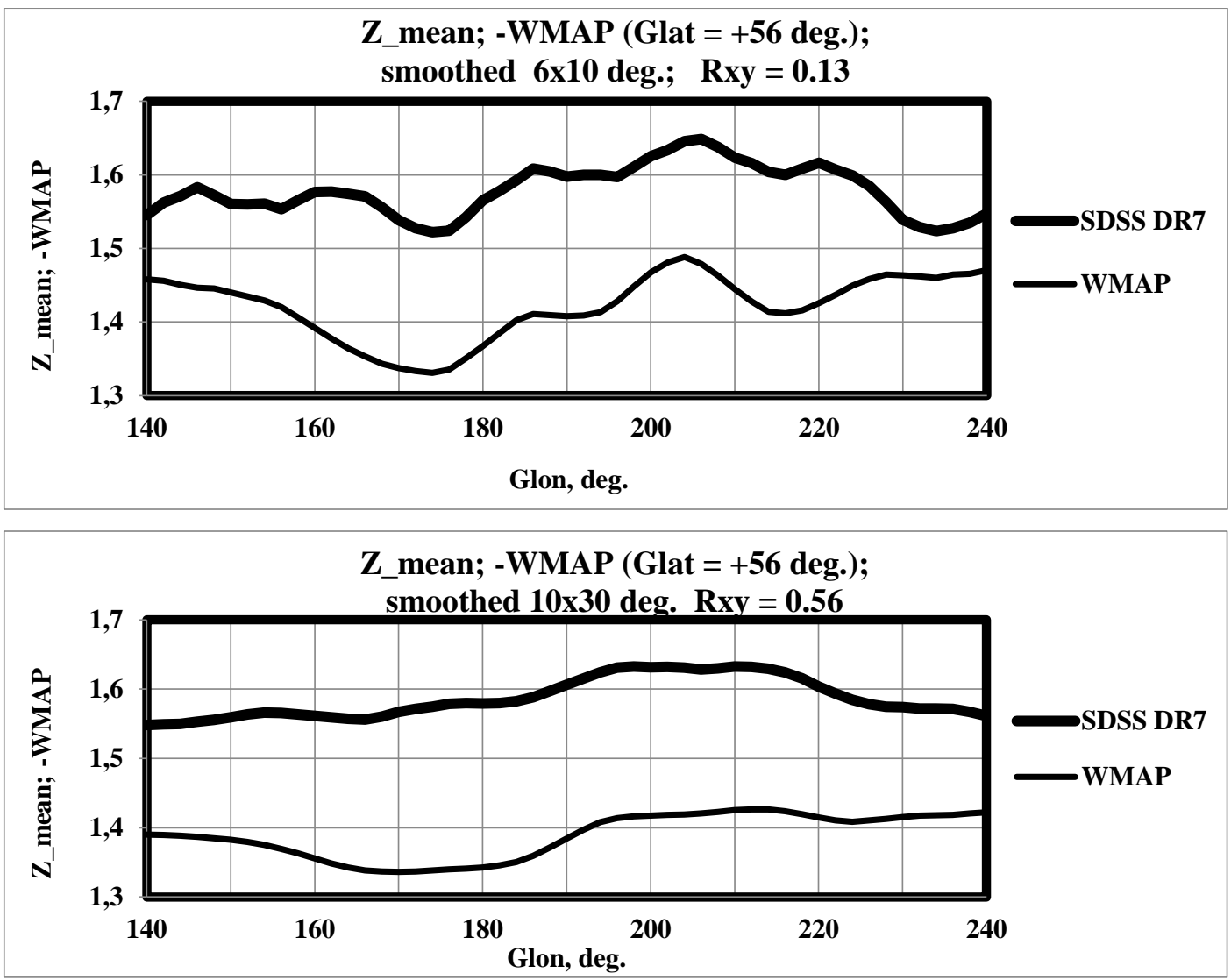

Fig. 5. Results of the comparison of the graphs of distribution of the compared values by the axis Glon at Glat $=56^{0}$ for different initial curves smoothing modes.

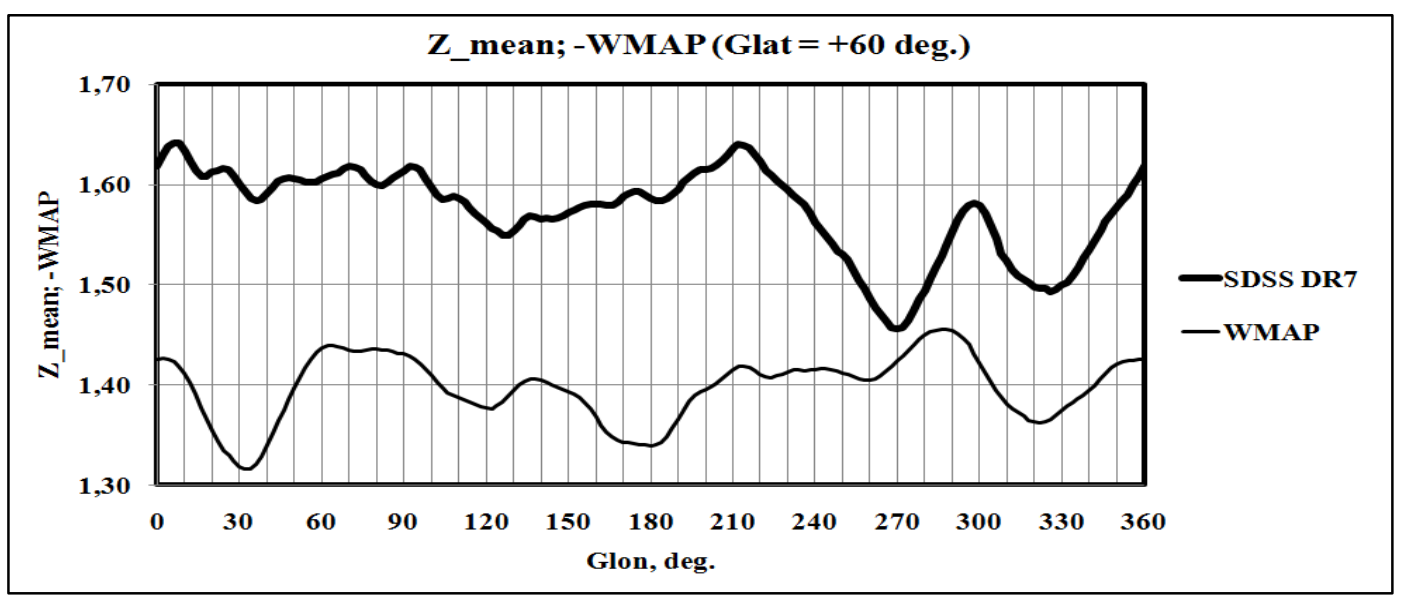




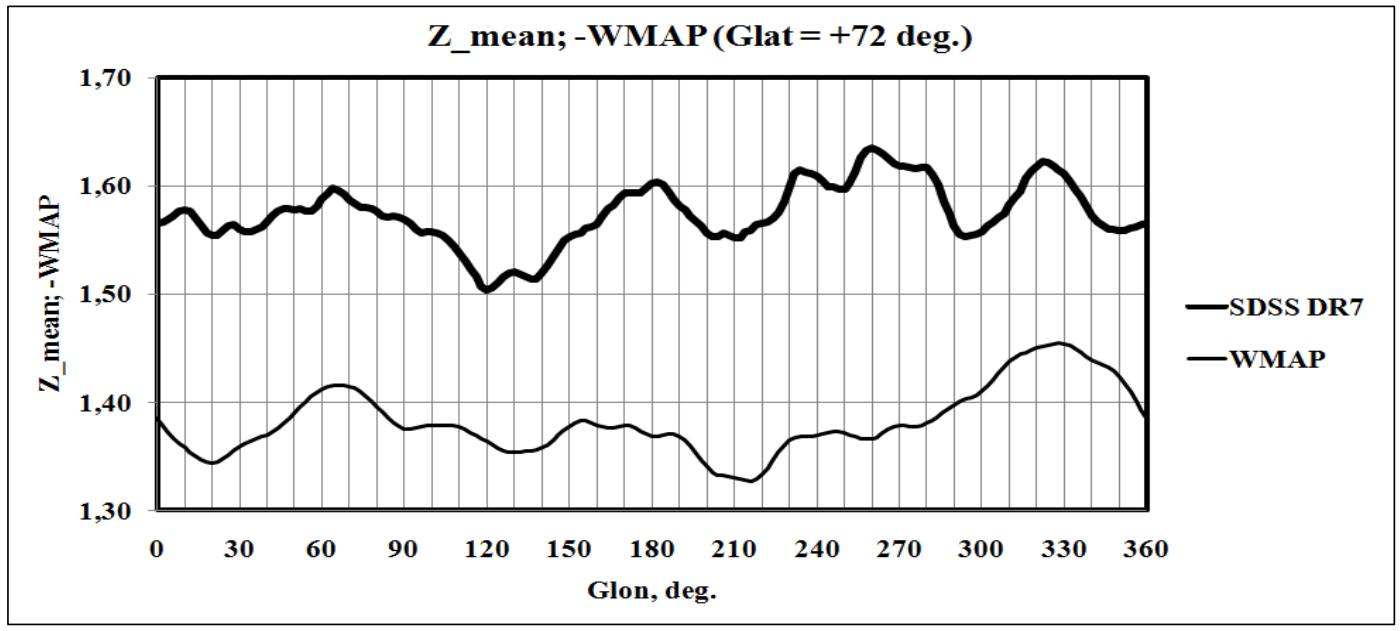

Fig. 6. Results of comparison of the distribution graphs of the compared values by the axis Glon at Glat $>58^{0}$. The smoothing mode is $10^{\circ} \times 30^{\circ}$.

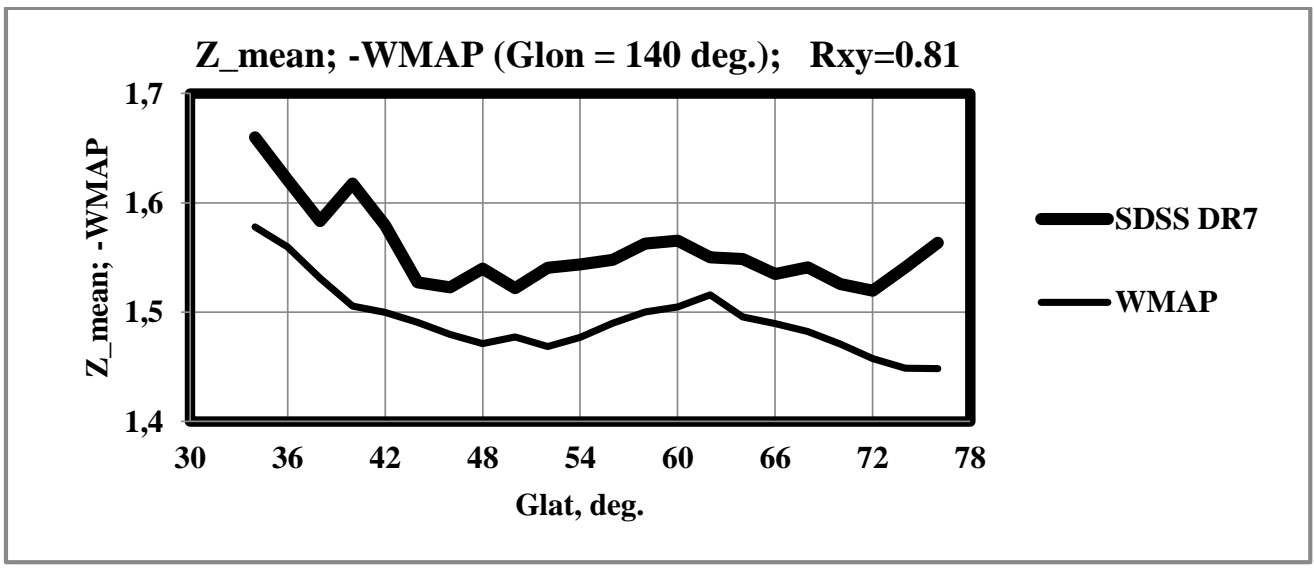

Fig. 7. Results of the comparison of the distribution graphs of the compared values by the axis Glat. The smoothing mode is $10^{0} \times 30^{\circ}$.

We should note that as we study the correlations of the distributions smoothed by the area sized $5 \times 15$ cells $\left(10^{0} \times 30^{0}\right)$, the shown correlation coefficients are related to the initial objects located in a wider interval of: $28^{0} \times 150^{0}(14 \times 75$ cells $)$.

Values of Rxy, obtained for the areas of different sizes and locations are shown in Table 2. 


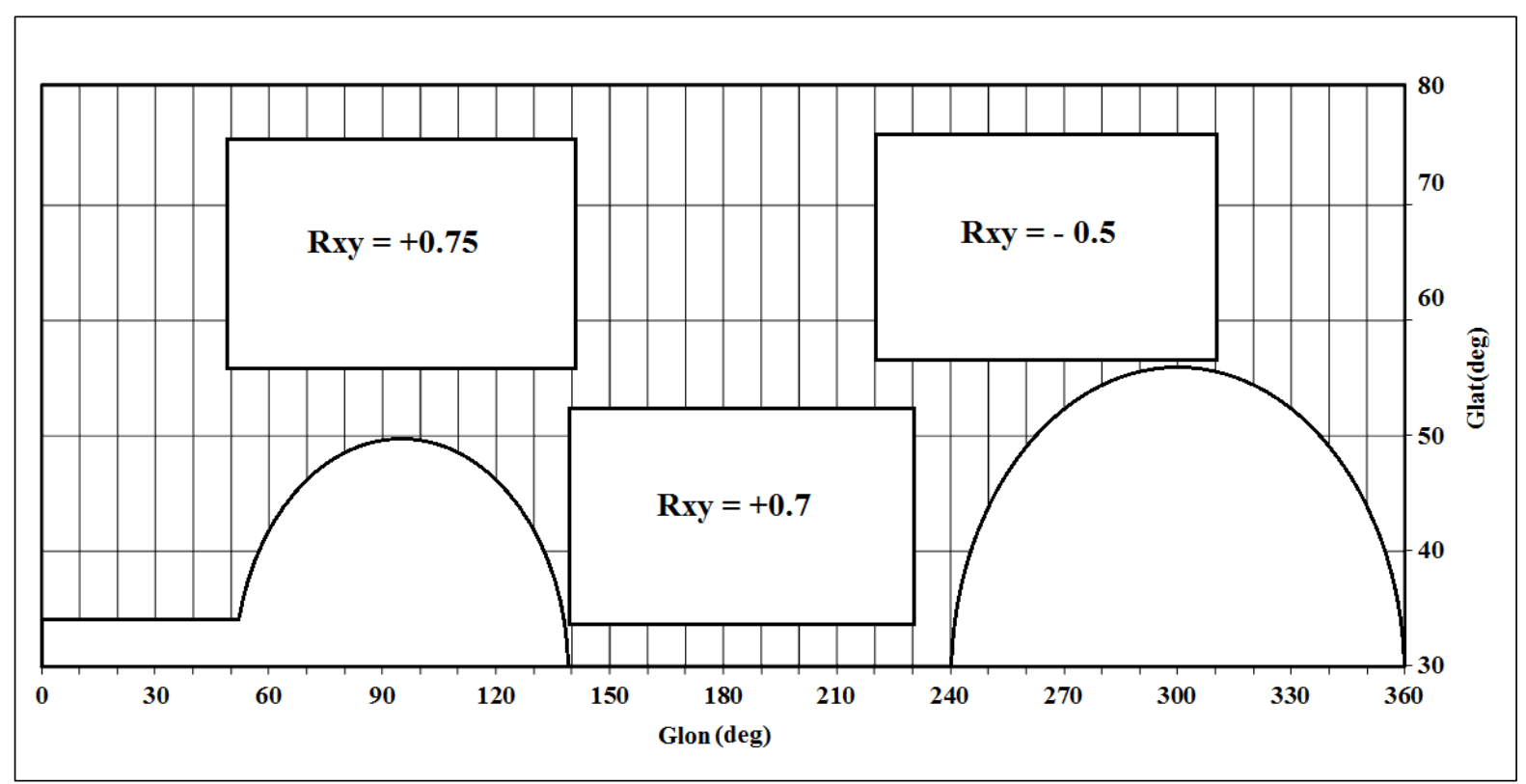

Fig. 8. Location of the rectangular sections sized $18^{0} \times 90^{0}(9 \times 45$ cells) with maximal by module values of Rxy in the area available for comparison.

Table 2. Values of Rxy for different smoothing parameters and different sizes and locations of the areas with positive correlation of the distributions

\begin{tabular}{|c|c|c|c|c|}
\hline $\begin{array}{l}\text { Smoothing if the initial } \\
\text { distributions on the } \\
\text { rectangle (Glon;Glat) }\end{array}$ & $\begin{array}{c}\text { Sizes of the area } \\
\text { of smoothed } \\
\text { distributions } \\
\text { (Glon;Glat) }\end{array}$ & $\begin{array}{c}\text { Coordinates of } \\
\text { the area center } \\
\text { (Glon;Glat) }\end{array}$ & $\begin{array}{l}\text { Sizes of the area of initial } \\
\text { objects (Glon;Glat) } \\
\text { with smoothing considered }\end{array}$ & $\begin{array}{c}\text { Value of Pearson } \\
\text { correlation } \\
\text { coefficient } \\
\text { Rxy }\end{array}$ \\
\hline \multirow{6}{*}{$10^{0} \times 6^{0}$} & $60^{0} \times 10^{0}$ & $70^{0} \times 66^{0}$ & $70^{0} \times 16^{0}$ & +0.56 \\
\hline & $60^{0} \times 10^{0}$ & $160^{0} \times 58^{0}$ & $70^{0} \times 16^{0}$ & +0.72 \\
\hline & $90^{0} \times 10^{0}$ & $90^{0} \times 70^{0}$ & $100^{0} \times 16^{0}$ & +0.48 \\
\hline & $90^{0} \times 10^{0}$ & $180^{0} \times 50^{0}$ & $100^{0} \times 16^{0}$ & +0.54 \\
\hline & $90^{0} \times 18^{0}$ & $90^{0} \times 66^{0}$ & $100^{0} \times 24^{0}$ & +0.45 \\
\hline & $90^{0} \times 18^{0}$ & $180^{0} \times 44^{0}$ & $100^{0} \times 24^{0}$ & +0.48 \\
\hline \multirow{5}{*}{$30^{0} \times 10^{0}$} & $60^{0} \times 18^{0}$ & $105^{0} \times 66^{0}$ & $90^{0} \times 28^{0}$ & +0.79 \\
\hline & $60^{0} \times 18^{0}$ & $185^{0} \times 44^{0}$ & $90^{0} \times 28^{0}$ & +0.80 \\
\hline & $90^{0} \times 18^{0}$ & $90^{0} \times 66^{0}$ & $120^{0} \times 28^{0}$ & +0.75 \\
\hline & $90^{0} \times 18^{0}$ & $175^{0} \times 44^{0}$ & $120^{0} \times 28^{0}$ & +0.70 \\
\hline & $120^{0} \times 18^{0}$ & $110^{0} \times 66^{0}$ & $150^{0} \times 28^{0}$ & +0.64 \\
\hline
\end{tabular}


A wide area of the negative values of Rxy can be related to the high tangential velocities of the big masses of matter which lead to the big angular shifts of the quasars distributions in relation to the initial distribution WMAP.

\section{Evaluation of the validity of the correlation coefficients}

To evaluate the statistical validity of the Pearson correlation coefficient data shown above we performed a simulation of random distribution of the compared values replacing their real values with numbers generated by RAND Excel 2007 and then further processing identical to the one described earlier. For the rectangles sized $18^{0} \times 90^{0}(9 \times 45$ cells) after approximately 100 thousands trials we obtained the distribution of probabilities of values of Rxy, close to the normal distribution with standard deviation of 0.28 .

For the real values in the area suitable for comparable analysis it appeared possible to obtain the values Rxy data for 1100 different positions of the rectangle with size $18^{0} \times 90^{0}$ (9x 45 cells). The results obtained for real and random values are shown on the Picture 9.

On the Picture 10 we show the correspondence of the probabilities for real and random values shown on the Picture 9, and probabilities of a random obtaining of real values, calculated by the formula of binominal distribution. For Rxy $>0.6$ these probabilities do not exceed $10^{-10}$, which confirms the validity of results shown in the previous chapter of this article.

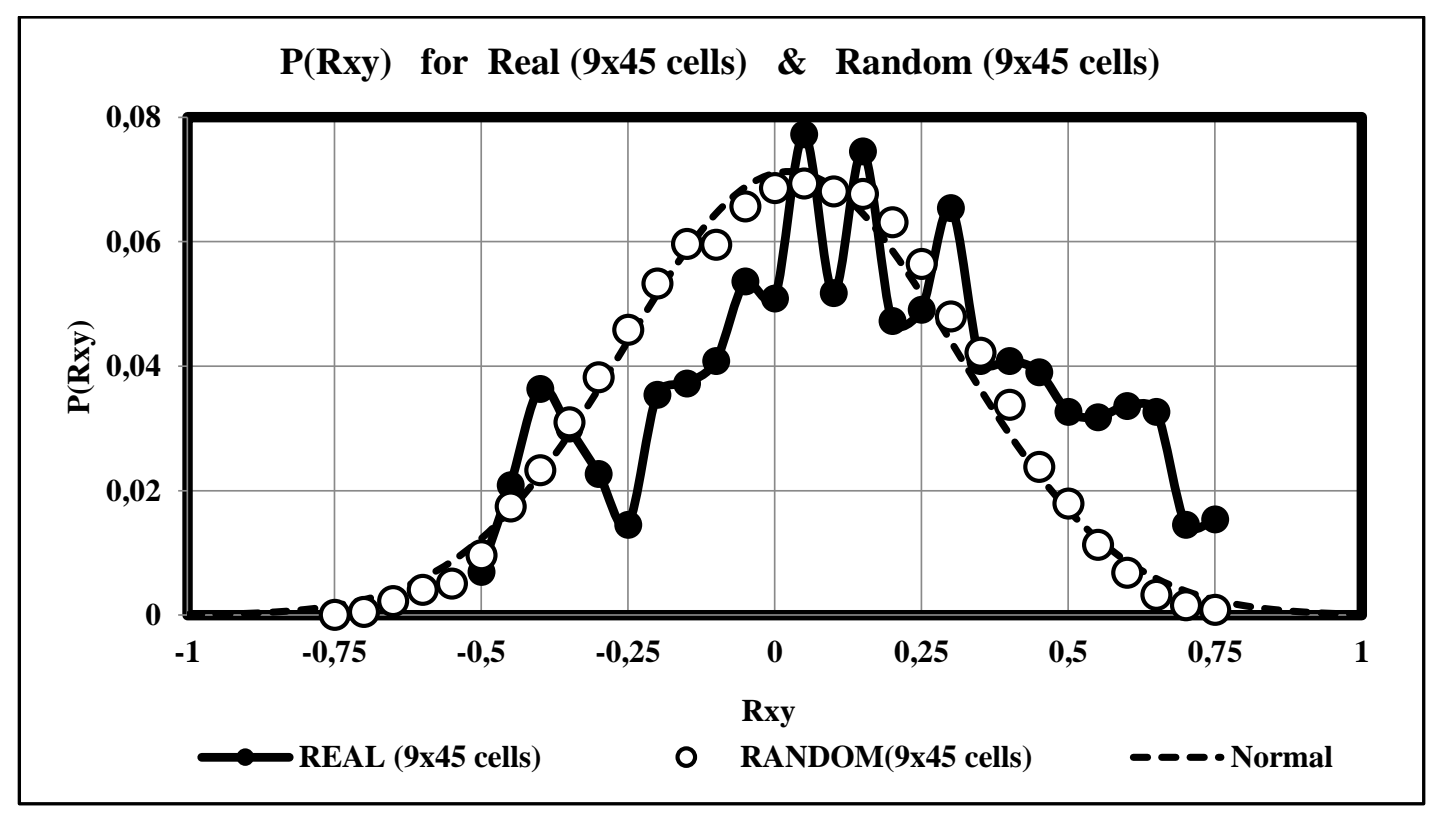

Fig. 9. Distribution of the probabilities of the values of Rxy for the real and random magnitudes. Smoothing mode $10^{0} \times 30^{0}$ 


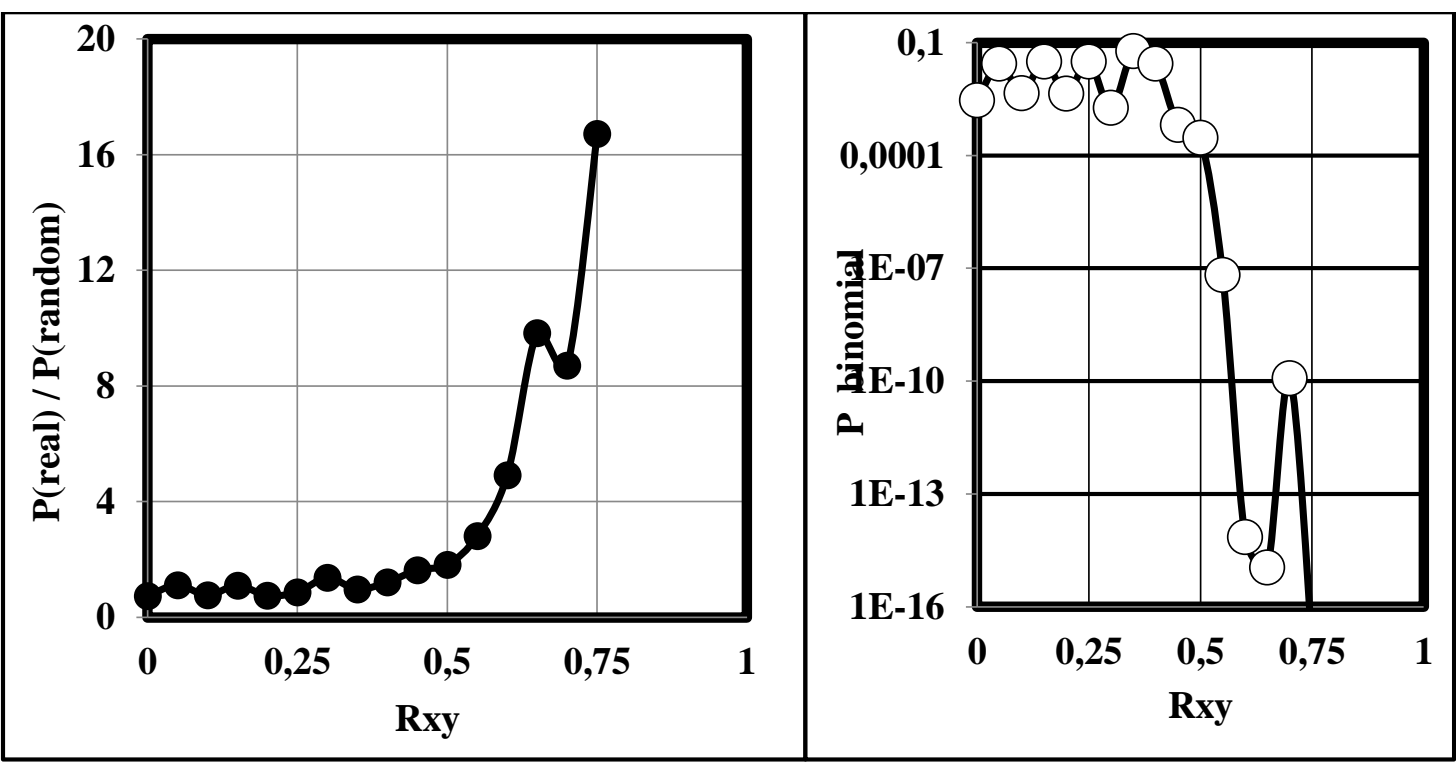

Fig. 10. Correspondence of the probabilities for the real and random magnitudes shown on the Picture 9, and the probability of the random obtaining of the real magnitudes calculated by the formula of binominal distribution.

This way, as a result of the performed in this article comparative analysis of the distributions -Asymm(Glon;Glat) and Zmean(Glon;Glat) in an area of the celestial sphere available for comparison, we discovered visual signs of a large-scale correlation and wide areas with credibly high values of Pearson correlation coefficient, which allows us to state the existence of the correlation between the angular distributions of the antisymmetrical component of the microwave backgrounds temperature deviation and the average values of redshifts of quasars.

\section{Comparison of the distributions of the average values of redshifts of quasars on the opposite areas of the celestial sphere}

The presented above results confirm the validity of the approach described in the chapter 2. But as we observe the sought correlation, the average values of the redshift of quasars are really calculated for the quasars distributed by all possible values of the distances, i.e. all the periphery of the big circle of the closed Universe.

At that, there appears an additional possibility to check the validity or invalidity of the studied model. If in some chosen point of the celestial sphere we can observe a set of objects seen all the way down the Universe, then in the opposite point of the celestial sphere we will see the same set of objects but located in inverted sequence by the distance. And if in the initial point the 
average value of the redshift and, correspondingly, the average remoteness of the objects decreases (has less than average value), we can expect that in the opposite point it would increase (have more than average value), as the average distance in it will be defined by the average distance for the initial point deducted from the length of circumference of the Universe.

To check this assumption we should chose rather wide opposite areas of the celestial sphere. This seems to be more comfortable in the coordinates in the terms RAJ2000; DEJ2000. In these coordinates we can chose two pairs of rather wide opposite areas of the celestial sphere containing a big amount of the observed quasars suitable for the comparative analysis:

№ 1: $\left(-3^{0}<\mathrm{DE}<3^{0} ; 120^{0}<\mathrm{RA}<240^{0}\right) \&\left(-3^{0}<\mathrm{DE}<3^{0} ;-60^{0}<\mathrm{RA}<60^{0}\right) . \quad 6968 \& 9568$ Quasars.

№ 2: $\left(5^{0}<\mathrm{DE}<11^{0} ; 120^{\circ}<\mathrm{RA}<240^{\circ}\right) \&\left(-11^{0}<\mathrm{DE}<-5^{0} ;-60^{0}<\mathrm{RA}<60^{\circ}\right) .8785 \& 3841$ Quasars.

For each of these four areas we calculated the average value of the redshift of quasars located within the cells sized $5^{0}(\mathrm{RA}) \times 6^{0}(\mathrm{DE})$, where the amount of quasars varied from 20 to 585 , around 300 quasars per cell in average.

On the Picture 11 we show the distribution of the average values of the redshift Zmean for the area 1. The high noise component does not let us make the comparative analysis of the obtained distributions. Because of that we performed the triple smoothing of the obtained curves by successive averaging by 5 , then by 3 and then again by 3 points. The pairs of smoothed distributions obtained as the result of it are shown on the Picture 12.

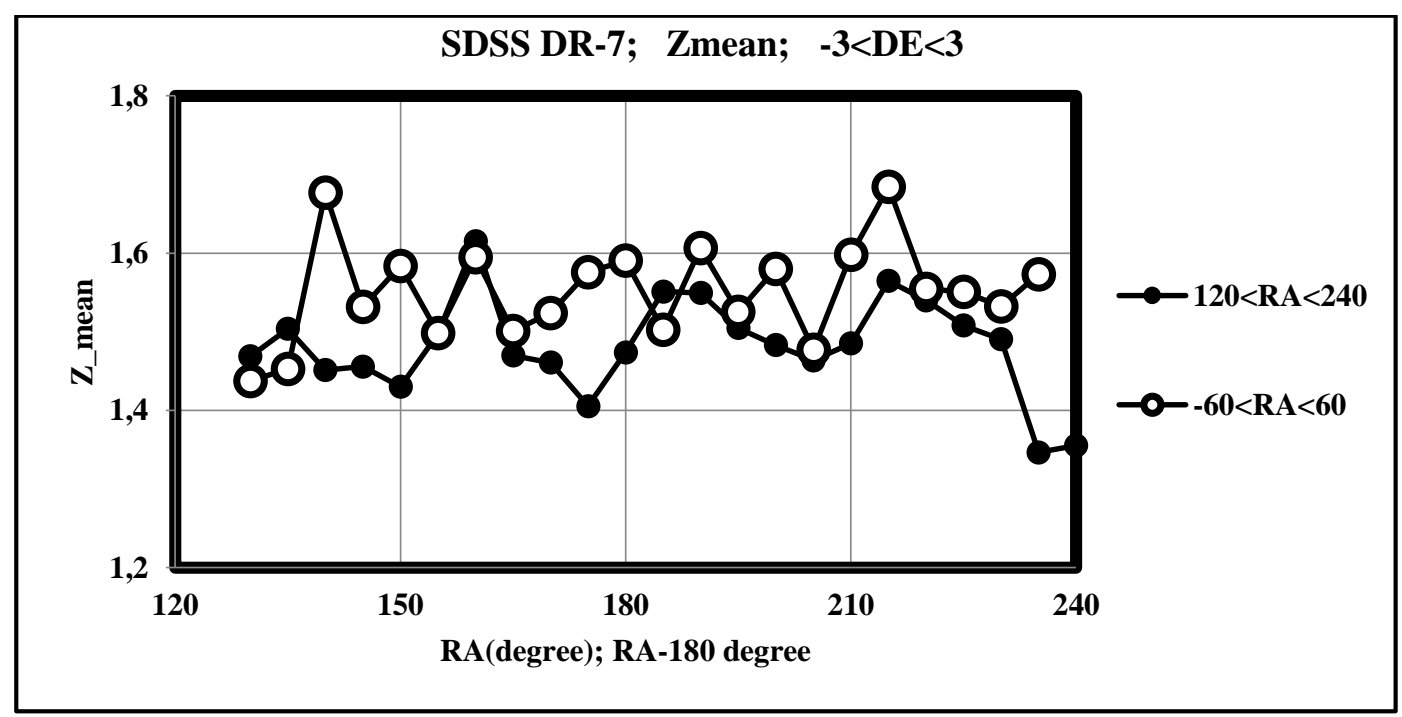


Fig. 11. Distribution along the axis RA of the values of the redshift of quasars from the SDSS-

DR7 catalog, averaged by the cells $5^{0} \times 6^{0}$, for the area 1 .
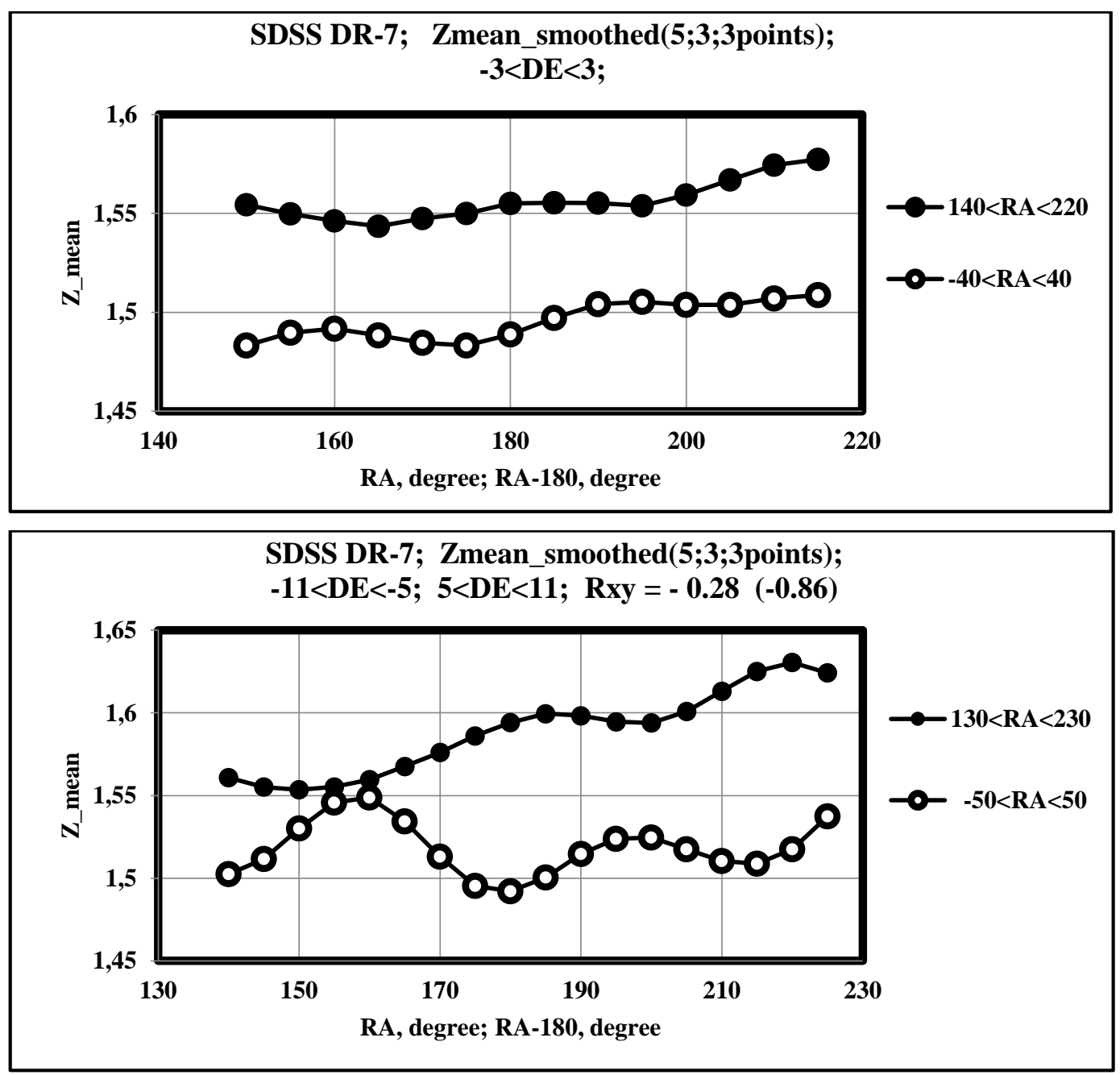

Fig. 12. Distribution of the average values of the redshift Zmean for the areas 1 and 2 after triple smoothing (according to the data from SDSS-DR7 catalog).

For the shown pairs of curves we calculated Pearson correlation coefficients. For area 1 it appeared to be above zero, supposedly because of the inclination of the curves, concealing the weak influence of the oppositely directed changes. To check this assumption, the inclination of curves was compensated, which led to the change of Rxy to the negative values direction (not shown here).

For the area 2 the opposite nature of the smoothed curves change appeared to be much more defined than for the area 1. Pearson correlation coefficient for this pair of curves is -0.28 . At compensation of the inclination it also changes towards more negative values reaching value -0.86 . 


\section{Conclusion}

We checked the assumption of a possible correlation of the microwave background inhomogeneities and the redshifts of quasars, and also of the negative correlation of the average values of the redshifts of quasars on the opposite areas of the celestial sphere.

The obtained results show the validity of our assumptions. We discovered the positive correlation of the antisymmetrical component of the microwave background temperature deviation distributions with the angular distributions of the average values of redshift of the quasars from the SDSS-DR7 catalog on the areas of the celestial sphere extending up to 120 degrees with Pearson correlation coefficients up to +0.75 . The negative correlation of the angular distributions of the average values of the redshifts of quasars on the opposite areas of the celestial sphere is noted on both available for comparison pairs of areas of the celestial sphere going down to 90 degrees with Pearson correlation coefficient values reaching -0.59 .

The shown data argues for the necessity to make a more thorough research of the phenomenon of the celestial sphere central symmetry and the conclusions about the dynamics of the development of the Universe resulting from it.

\section{References}

1. Land K., Magueijo J. (2005). Examination of Evidence for a Preferred Axis in the Cosmic Radiation Anisotropy. Phys. Rev. Lett, 95, 071301.

2. Kudriavtcev I., Semenov D.A. (2010). Central symmetry and antisymmetry of the microwave background inhomogeneities on Wilkinson Microwave Anisotropy Probe maps. arXiv, 1008.4085 .

3. Kudriavtcev I. (2010). Manifestation of central symmetry of the celestial sphere in the mutual disposition and luminosity of the Quasars. arXiv, 1009.4424.

4. Kudriavtcev I. (2012). On inner contradiction in the metric tensor of the standard cosmological model. Physical Interpretation of Relativity Theory: Proceedings of International Meeting. Moscow,4-7 July 2011, Moscow: BMSTU, 178-185.

5. Kudriavtcev I. Specific features of the average magnitudes and luminosities of quasars and galaxies as a function of redshift and their interpretation in the modified cosmological model. arXiv, 1109.3630. 
Proceedings of International Conference PIRT-2015

6. The Sloan Digital Sky Survey quasar catalog (2010). Retrieved from: http://vizier.ustrasbg.fr/viz-bin/VizieR?-source=VII\%2F260

7. Internal Linear Combination Maps. (2010). Retrieved from:

http://lambda.gsfc.nasa.gov/product/map/current/m_images.cfm 